\title{
Propiedades ópticas y térmicas de una oligoimina conjugada con terminación indol
}

\author{
Francisco G. Mejía a*, Rosa A. Vázquez ${ }^{a}$, Karina A. Ayala ${ }^{\mathrm{b}}$, Armando I. Martínez ${ }^{\mathrm{c}}$, Gerardo V. García ${ }^{\mathrm{c}}$. \\ a Área Académica de Ciencias de la Tierra y Materiales, Universidad Autónoma del Estado de Hidalgo, Hidalgo, México. \\ b Área Académica de Computación y Electrónica, Universidad Autónoma del Estado de Hidalgo, Hidalgo, México. \\ c Área Ingeniería Mecánica Automotriz, Universidad Politécnica de Pachuca, Zempoala, Hidalgo, México.
}

\section{Resumen}

En este trabajo se realizó la comparativa sobre las propiedades ópticas y térmicas de un compuesto orgánico, una oligoimina conjugada con características semiconductoras, con potencial aplicación en optoelectrónica, sintetizando y caracterizando como partida un pentámero conjugado para posteriormente agregar a este las terminaciones de tipo indol, en busca de mejorar sus propiedades ópticas y como donador de electrones. La síntesis se llevó a cabo por la ruta de mecanosíntesis en un tiempo de 90 minutos, sin la utilización de disolventes ni catalizadores mediante el uso de un molino de alta energía.

Palabras Clave: iminas, semiconductores, mecanosíntesis, optoelectrónica, OPV.

\section{Introducción}

Los semiconductores orgánicos tienen aplicaciones prácticas en diferentes dispositivos optoelectrónicos tales como las celdas solares orgánicas (OPV's), diodos emisores de luz orgánicos (OLED's), ventanas inteligentes, transistores de efecto de campo orgánicos (OFET’s), transistores de película delgada (TTF's) entre otros.

Actualmente el desarrollo de la tecnología sobre la optoelectrónica ha promovido la búsqueda de nuevos semiconductores orgánicos con las características adecuadas para mejorar su desempeño y eficiencia al ser evaluados en los dispositivos.

En el grupo de trabajo del laboratorio de polímeros del Área Académica de Ciencias de la Tierra y Materiales de la Universidad Autónoma del Estado de Hidalgo se ha buscado desarrollar nuevos materiales semiconductores orgánicos, dicho grupo de trabajo (Martínez et al.) logró sintetizar una familia de productos semiconductores del tipo imina, a partir de esto, en el presente trabajo se propuso la síntesis del compuesto denominado OIC1-I, el cual tiene una estructura conjugada de tipo imina con grupos terminales indol, lo que permite incrementar su capacidad como donador de electrones.

Para la obtención de OIC1-I se aplicó la ruta de mecanosíntesis en dos etapas, la primera para la obtención del centro OIC1, que fue validada por las técnicas espectroscópicas de FTIR (Infrarrojo con Transformada de Fourier) y $\mathrm{RMN}^{1} \mathrm{H}$ (Resonancia Magnética Nuclear de Protón), comprobando que su estructura contara con las terminaciones amino. En la segunda etapa se llevó a cabo la incorporación de los grupos terminales indol para así obtener a OIC1-I, al cual se le aplicaron nuevamente las técnicas de FTIR y $\mathrm{RMN}^{1} \mathrm{H}$ para verificar la formación de enlace $\mathrm{C}=\mathrm{N}$ entre el centro $\mathrm{OIC} 1$ y el grupo terminal.
Convirtiendo a OIC1-I en candidato para ser aplicado como capa activa en una celda solar orgánica, la cual será capaz de transformar la energía solar en energía eléctrica por el fenómeno del efecto fotovoltaico.

Las condiciones establecidas en la ruta de mecanosíntesis permitieron llevar a cabo la reacción para la formación del enlace imina $(\mathrm{C}=\mathrm{N})$ en fase sólida en un periodo de 90 minutos en ausencia de catalizadores y disolventes en cada una de las etapas, el cual se puede considerar un periodo relativamente corto comparado con las 24 horas requeridas por la ruta de síntesis convencional. Con base a las características que ofrece la ruta de mecanosíntesis para la obtención de semiconductores orgánicos de tipo imina, en este caso de OIC1-I, se puede considerar como una alternativa viable, efectiva, económica y ecológica.

\section{Síntesis y elucidación estructural}

La síntesis de los compuestos se realizó por la ruta de mecanosíntesis en un molino de alta energía, durante un tiempo de 90 minutos para cada reacción y en ausencia de disolventes y catalizadores. El primer compuesto, que se toma de partida, OIC1, es un pentámero con terminaciones $\mathrm{NH} 2$ sintetizado con reactivos 2,5-bis(octiloxi) tereftaldehido y 1,4fenilendiamina.

El segundo compuesto es el formado a partir de OIC1 funcionalizado con el grupo indol-3-carboxaldehido, resultando un heptámero con cadena principal conjugada con enlaces del tipo imino.

\footnotetext{
* Autor en correspondencia.

Correo electrónico: fco.german.mejia@gmail.com (Francisco G. Mejía)
} 


\subsection{Síntesis de un pentámero $(\mathrm{OICl})$}

La síntesis del compuesto OIC1 (Figura 1) se realizó a través de la ruta de mecanosíntesis.

La reacción de condensación se llevó a cabo entre dialdehidos y diaminas dentro de un contenedor de acero inoxidable con seis bolas también de acero inoxidable de $1 \mathrm{~cm}$ de diámetro en un molino de alta energía SPEX 8000D durante un tiempo de 90 minutos en ausencia de disolventes y catalizadores. La ruta de mecanosíntesis ofrece las bondades de reducir el tiempo de reacción a 90 minutos con respecto al tiempo de reacción convencional de 24 horas y que no se requiere de disolventes ni catalizadores para que se produzca la reacción.

El agua producida al realizarse la reacción de condensación se retiene en un filtrado con sulfato de sodio anhidro, así como también los residuos de hierro desprendido del contenedor y las bolas de acero inoxidable durante la molienda.

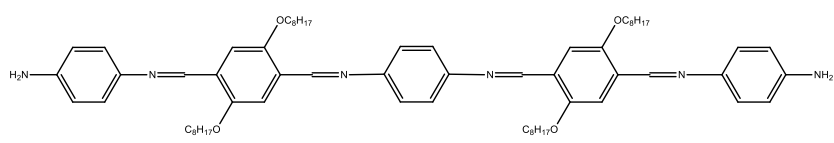

Figura 1: Compuesto orgánico conjugado de tipo imina denominado OIC1, con terminaciones $\mathrm{NH}_{2}$ que posibilita la adición del grupo indol.

\subsection{Sintesis de un heptámero (OICl-I)}

La síntesis del compuesto denominado OIC1-I, es un heptámero producido a partir de la molécula del compuesto OIC1 en estado sólido adicionando el reactivo indol-3carboxaldehido, la molienda se realiza durante 90 minutos en el molino de alta energía. La molécula del compuesto OIC1-I se muestra a continuación (Figura 2).

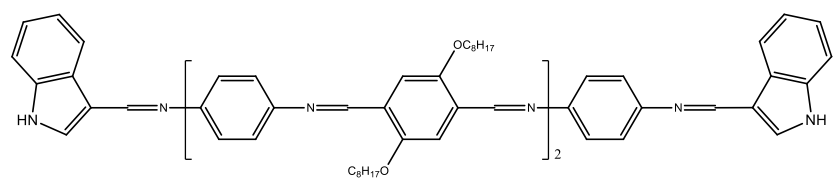

Figura 2: Compuesto orgánico conjugado de tipo imina denominado OIC1-I, con terminaciones de indol.

Se observa la conjugación de los enlaces, característico de los compuestos orgánicos semiconductores. El filtrado se realiza de la misma manera para retener el agua y el hierro desprendido durante la reacción.

El espectro de FTIR muestra las bandas correspondientes a las vibraciones de los grupos funcionales; se observa en 3350 cm-1 la banda de vibración de tensión de los grupos $\mathrm{NH}$, las bandas de vibración de los grupos (C-H) de los anillos aromáticos se distinguen a $3047 \mathrm{~cm}-1$, en $1647 \mathrm{~cm}-1$ aparece una banda que corresponde a la vibración de tensión (v) característica del grupo imino $(\mathrm{C}=\mathrm{N})$ que confirma la formación de la oligoimina, en $1610 \mathrm{~cm}-1$ se presenta la banda asignada al grupo (-C=C-) en el modo de vibración de tensión del esqueleto conjugado, las bandas a 2952, 2925 y $2855 \mathrm{~cm}-1$ corresponden a la vibración de los enlaces $(\mathrm{CH})$ de los grupos alifáticos metilenos $(\mathrm{CH} 2)$ y metilos $(\mathrm{CH} 3)$.

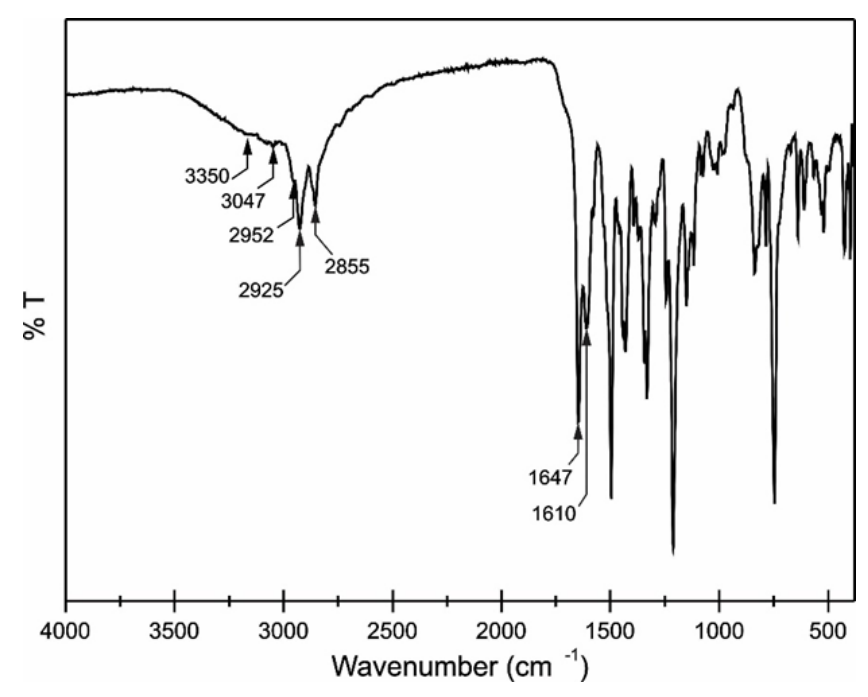

Figura 3: Compuesto orgánico conjugado de tipo imina denominado OIC1-I, con terminaciones de indol.

La técnica espectroscópica de FTIR nos muestra la obtención del producto, sin embargo, la técnica espectroscópica de $\mathrm{RMN}^{1} \mathrm{H}$ nos confirma plenamente la obtención del compuesto.

En la figura 4 se muestra la molécula con los índices asignados a las señales del espectro de Resonancia Magnética Nuclear de Protón RMN-1H de OIC1-I (Figura 5) donde se observa una señal que integra para dos protones a $10.1 \mathrm{ppm}$ correspondiente al segmento NH del grupo indol, en la región que va de 9.1 a $8.4 \mathrm{ppm}$ se encuentran las señales que corresponden a los protones de los grupos imino e integran para seis protones, en la región que va de 7.9 a 7.3 a ppm se encuentran las señales que corresponden a los protones de los grupos aromáticos e integran para 24 protones, a $4.2 \mathrm{ppm}$ se encuentra una señal triple que corresponde a los protones de los grupos metileno alfa al oxígeno de la cadena alquiloxi e integra para ocho protones, en la región que va de 2 a 1 ppm se encuentran las señales que corresponden a los protones de los grupos metileno restantes de la cadena metiloxi e integran para 48 protones, finalmente a $0.8 \mathrm{ppm}$ se encuentra la señal que corresponde a los protones de los grupos metilo de las cadenas alquiloxi e integra para doce protones.

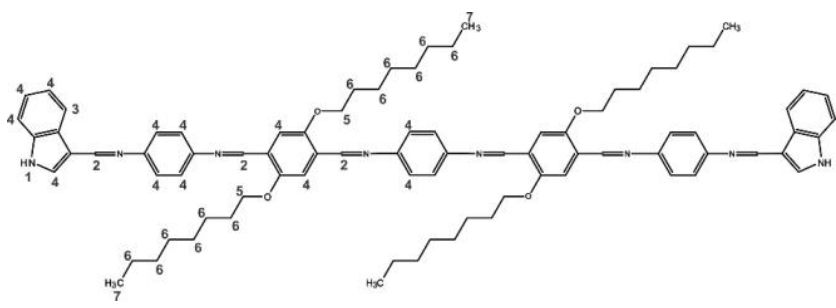

Figura 4: Compuesto OIC1-I, con los índices asignados a las señales del espectro de Resonancia Magnética Nuclear de Protón. 


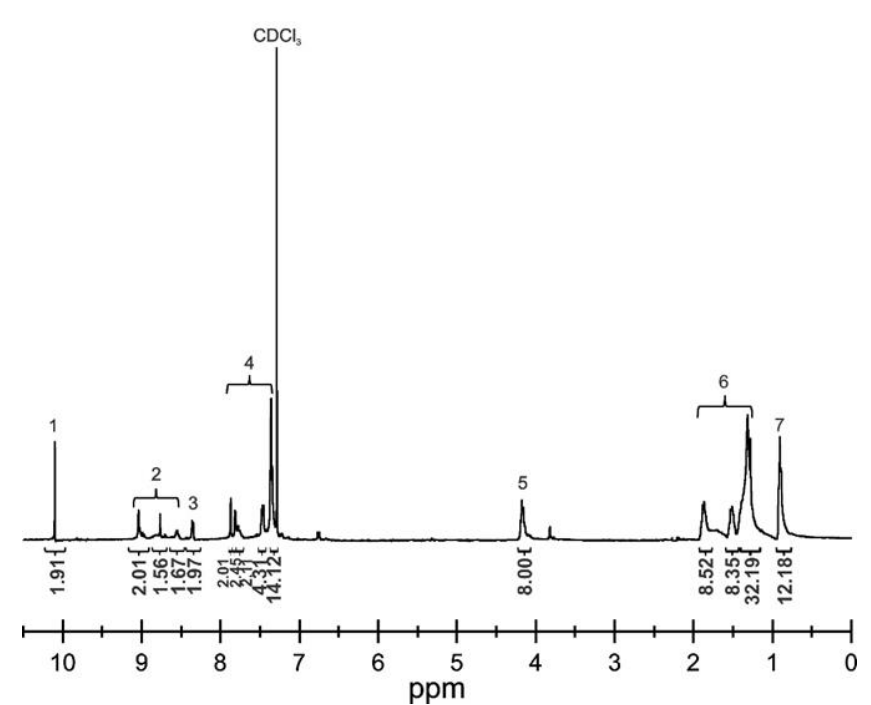

Figura 5: Espectro de $\mathrm{RMN}^{1} \mathrm{H}$ del compuesto OIC1-I.

\section{Caracterización óptica en solución}

La caracterización óptica en solución del compuesto OIC1, se realizó con disolvente THF y se obtuvieron espectros de absorbancia y emisión. En la tabla siguiente se presentan los resultados obtenidos.

Tabla 1: Resumen de propiedades ópticas de OIC1

\begin{tabular}{ccccc}
\hline Compuesto & $\begin{array}{c}\lambda_{\text {Abs max }} \\
(\mathbf{n m})\end{array}$ & $\begin{array}{c}\lambda_{\text {Emi max }} \\
(\mathbf{n m})\end{array}$ & $\begin{array}{c}\mathbf{E g}^{\text {opt }} \\
(\mathbf{e V})\end{array}$ & $\begin{array}{c}\text { Desplazamiento } \\
\text { Stokes (nm) }\end{array}$ \\
\hline OIC1 & 438 & 520 & 2.39 & 82 \\
\hline
\end{tabular}

El espectro de absorbancia y emisión en solución del compuesto OIC1-I se obtuvo a partir de UV-Vis y fluorescencia (Figura 6). Los resultados mostraron una banda de absorción a $289 \mathrm{~nm}$ correspondiente a la transición $\pi-\pi^{*}$ de los núcleos aromáticos y una longitud máxima de absorción en el espectro visible de $433 \mathrm{~nm}$ correspondiente a la transición $\mathrm{n}-\pi^{*}$ de los grupos $\mathrm{C}=\mathrm{N}$ conjugados, también se determinó un band gap óptico en solución de $2.4 \mathrm{eV}$ aproximadamente y un desplazamiento de Stokes de $90 \mathrm{~nm}$ que revela la pérdida de energía no radiativa entre niveles vibracionales durante el proceso de excitación y emisión (Tabla 2).

Tabla 2: Resumen de propiedades ópticas de OIC1-I

\begin{tabular}{ccccc}
\hline Compuesto & $\begin{array}{c}\lambda_{\text {Abs max }} \\
(\mathbf{n m})\end{array}$ & $\begin{array}{c}\lambda_{\text {Emi max }} \\
(\mathbf{n m})\end{array}$ & $\begin{array}{c}\mathbf{E g}^{\text {opt }} \\
(\mathbf{e V})\end{array}$ & $\begin{array}{c}\text { Desplazamiento } \\
\text { Stokes }(\mathbf{n m})\end{array}$ \\
\hline OIC1-I & 433 & 523 & 2.4 & 90 \\
\hline
\end{tabular}

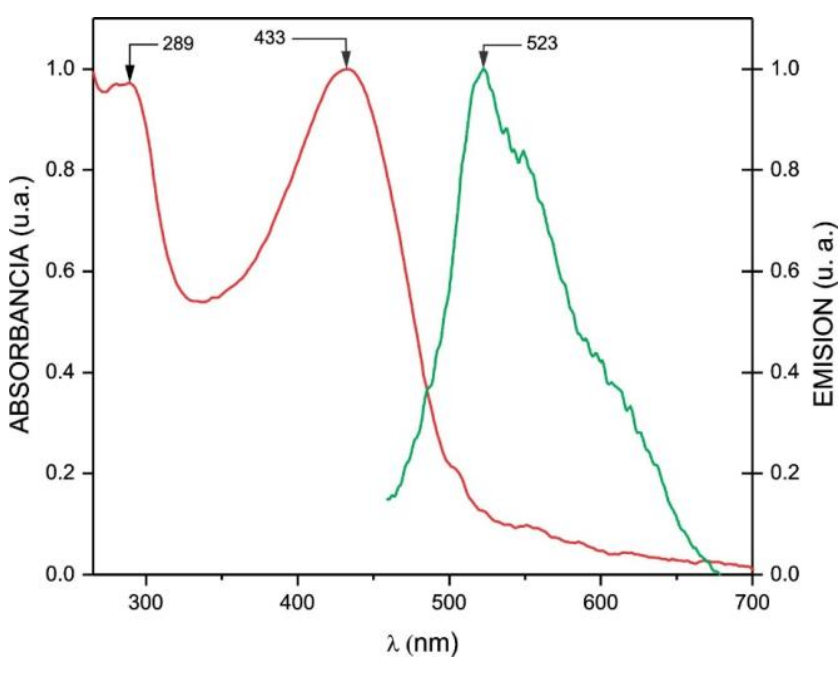

Figura 5: Espectro de $\mathrm{RMN}^{1} \mathrm{H}$ del compuesto OIC1-I.

\section{Caracterización térmica}

Para la caracterización térmica del compuesto OIC1 se hizo un Análisis Termogravimétrico (TGA) en atmósfera de nitrógeno, encontrando una temperatura de degradación de $138^{\circ} \mathrm{C}$.

Para el caso del compuesto OIC1-I el análisis termogravimétrico (Figura 6) mostró una temperatura de degradación de $192.7^{\circ} \mathrm{C}$

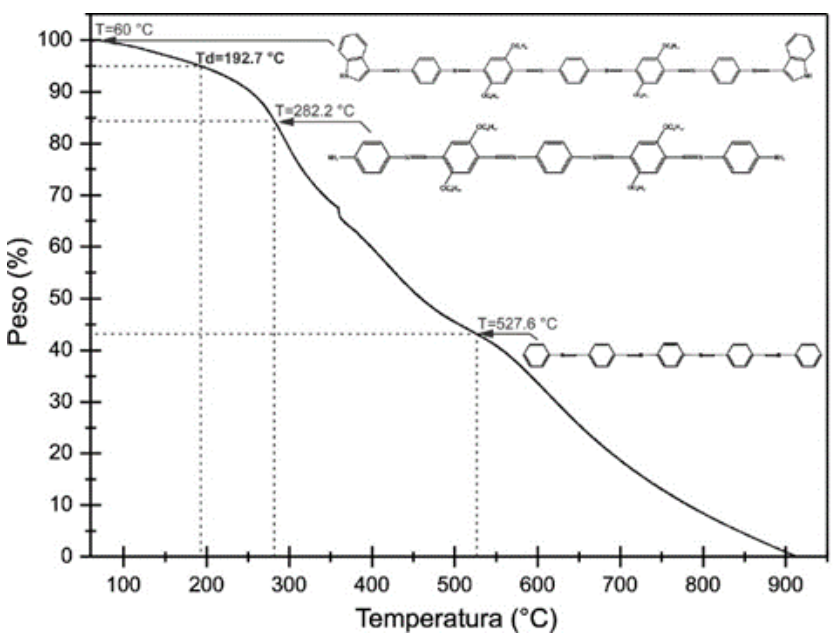

Figura 6: Termograma del compuesto OIC1-I.

La temperatura inicial de degradación (Td) del compuesto se presentó a $192.7^{\circ} \mathrm{C}$, además se observaron tres etapas de descomposición de OIC1-I, donde la primera etapa tuvo una pérdida de peso de $16 \%$ entre una temperatura de 60 a 282.2 ${ }^{\circ} \mathrm{C}$, la cual corresponde a la degradación del grupo terminal de tipo indol. La segunda etapa de degradación en el intervalo de 282.2 a $527.6{ }^{\circ} \mathrm{C}$ se presentó una pérdida de peso de aproximadamente $41 \%$ debido al grupo amina $\mathrm{NH} 2$ y a las cadenas alcoxi flexible. En la tercera etapa se observó una pérdida de peso del $43 \%$ en la región de 527.6 a $950{ }^{\circ} \mathrm{C}$, donde se degradaron los grupos aromáticos y los enlaces imina de la cadena principal dejando un $0 \%$ de carbón residual. 


\section{Conclusiones}

Las características ópticas en ambos compuestos, OIC1 y OIC1-I demuestran que son buenos candidatos para ser utilizados en dispositivos optoelectrónicos por sus características de semiconductores, en virtud de que ambos compuestos mantienen una banda de absorbancia y emisión dentro del espectro visible y cercano al verde y la brecha energética alrededor de los $2.4 \mathrm{eV}$ que los coloca dentro de los semiconductores orgánicos; el compuesto OIC1-I muestra una longitud de absorbancia máxima a 433nm, 5nm hacia longitudes de onda más cortas, sin embargo muestra una amplia banda de absorción que va de los $350 \mathrm{~nm}$ a los $500 \mathrm{~nm}$ aproximadamente. En el espectro de emisión de OIC1-I se observa una longitud de onda de máxima emisión de 523nm, 3 $\mathrm{nm}$ hacia longitudes de onda de menor energía que el compuesto OIC1. La brecha energética de ambos compuestos solo difiere por $0.01 \mathrm{eV}$, el compuesto OIC1-I muestra la mayor brecha energética y la energía de pérdida no radiativa es mayor en $8 \mathrm{~nm}$ para el compuesto OIC1-I.

De acuerdo a los resultados de los análisis termogravimétricos, ambos compuestos son buenos candidatos para ser utilizados en dispositivos optoelectónicos debido a que ambos presentan su temperatura de degradación por encima de $\operatorname{los} 105^{\circ} \mathrm{C}$.

Aunque los valores de band gap óptico y de la pérdida de energía no radiativa para el compuesto OIC1-I se muestran mayores, será necesario realizar una caracterización eléctrica y una evaluación de la eficiencia del desempeño en algún dispositivo de los antes mencionados, para establecer una caracterización más completa y determinar que tan bueno resulta para su utilización como semiconductor orgánico.

\section{English Summary}

Optical and thermal properties of an oligoimine conjugated with indole termination.

\section{Abstract}

In this work, a comparison was made on the optical and thermal properties of an organic compound, an oligoimine conjugated with semiconductor characteristics, with potential application in optoelectronics, synthesizing and characterizing a conjugated pentamer as starting material to later add to this the indole type terminations, in search of improving its optical properties and as an electron donor. The synthesis was carried out by the mechanosynthesis route in a time of 90 minutes, without the use of solvents or catalysts through the use of a high-energy mill.

Keywords:

imines, semiconductors, mechanosynthesis, optoelectronics, OPV

\section{Referencias}

Y. Shirota, 2013. Small molecular weight materials for (opto)electronics applications: overview, Woodhead Publishing.

Leznoff C., Lever A., (1992), Phthalocyanines, properties and applications, New York, VCH.

Hanack M, Dini D, Barthel M.,Vagin S., (2002), 'Conjugated macrocycles as active materials in nonlinear optical processes: optical limiting effect with phthalocyanines and related compounds', Chem Rec, 2 , 129-148.

Claessens C., Hahn U., Torres T., (2008), 'Phthalocyanines: from outstanding electronic properties to emerging applications', Chem Rec, 8, 75-97.

Wright J., (1995), Molecular crystals, 2nd edition, Cambridge, Cambriged University Press.

Akamatu H., Inokuchi H., (1950), 'On the electrical conductivity of violanthrone, iso-violanthrone, and pyranthrone', J Chem Phys, 18, 810-811.

Akamatu H., Inokuchi H., Matsunaga Y., (1954), 'Electrical conductivity of the perylene-bromine complex', Nature, 173, 168-169.

Shirota Y., Kobata T., Noma N., (1989), 'Starburst molecules for amorphous molecular materials. 4,4',4',-Tris(N,Ndiphenylamino)triphenylamine and 4,4',4' -tris[N-(3-methylphenyl)N-phenylamino]triphenylamine', Chem Lett,1145-1148.

Ito T., Shirakawa H., Ikeda S., (1974), 'Simultaneous polymerization and formation of polyacetylene film on the Surface of concentrated soluble Zeigler-type catalyst solution', J Polym Sci: Polym Chem Ed.12, 1120

Shirakawa H., Louis E. J., MacDiarmid A. G, Chiang C. K., Hegger A. J., (1977), 'Synthesis of electrically conducting organic polymers: hologen derivatives of polyacetylene, $(\mathrm{CH})$, , , J Chem Soc Chem Commun, 578-580.

Chiang C. K., Park Y. W., Heeger A. J., Shirakawa H., Louis E. J., MacDiarmid A. G., (1978), 'Conducting polymers: halogen doped polyacetylene', J Chem Phys, 69,5098-5104.

Skotheim T. A., (1998), Handbook of conducting polymers, Third Edition, New York, Marcel Dekker.

Kanega H., Shirota Y., Mikawa H., (1984), 'Electrochemical doping of poly(N-vinylcarbazole) and poly (3,6-dibromo-N-vinylcarbazole) and their electrical conductivities', J Chem Soc ChemCommun, 158-159.

Shirota Y., Noma N., Kanega H., Mikawa H., (1984), 'Preparation of an electrically conducting polymer by the electrolytic polymerization of N-vinylcarbazole', J Chem Soc Chem Commun, 470-471.

Shirota Y., Noma N., Shimizu Y., Kanega H., Jeon I-R, Nawa K., Kakuta T., Yasui H., Namba K., (1991), 'Preparation of electrically conducting polymers containing pendant $\pi$-electron systems by electrochemical doping, and properties and applications of doped polymer', Synth Met, 41-43, 3031-3036.

Strohriegl P., Grazulevicius J. V., (2002), 'Charge-transporting molecular glasses', Adv Mater, 14, 1439-1452.

Coropceanu V., Cornil J., da Silva Filho D. A., Olivier Y., Silbey R., Brédas J-L., (2007), 'Charge transport in organic semiconductors', Chem Rev, 107, 926-952.

Shirota Y., Kageyama H., (2007), 'Charge carrier transporting molecular materials and their applications in devices', Chem Rev,107, 953-1010.

Walzer K, Maennig B, Pfeiffer M and Leo K (2007), 'Highly efficient organic devices base don electrically doped transport layers', Chem Rev, 107, 1233-1271.

O’Brien D, Bleyer A, Lidzey D G, Bradley D D C and Tsutsui T (1997), 'Efficient multilayer electroluminescence devices with poly(mphenylenevinylene-co-2,5-dioctyloxy-p-phenylenevinylene) as the emissive layer', J Apll Phys, 82, 2662-2670.

Armando I. Martínez, 'Mecanosíntesis y síntesis convencional de nuevos sistemas conjugados de tipo oligofenileniminas y estudios de sus propiedades para potencial aplicación en la optoelectrónica', Tesis doctoral, UAEH, 2012 